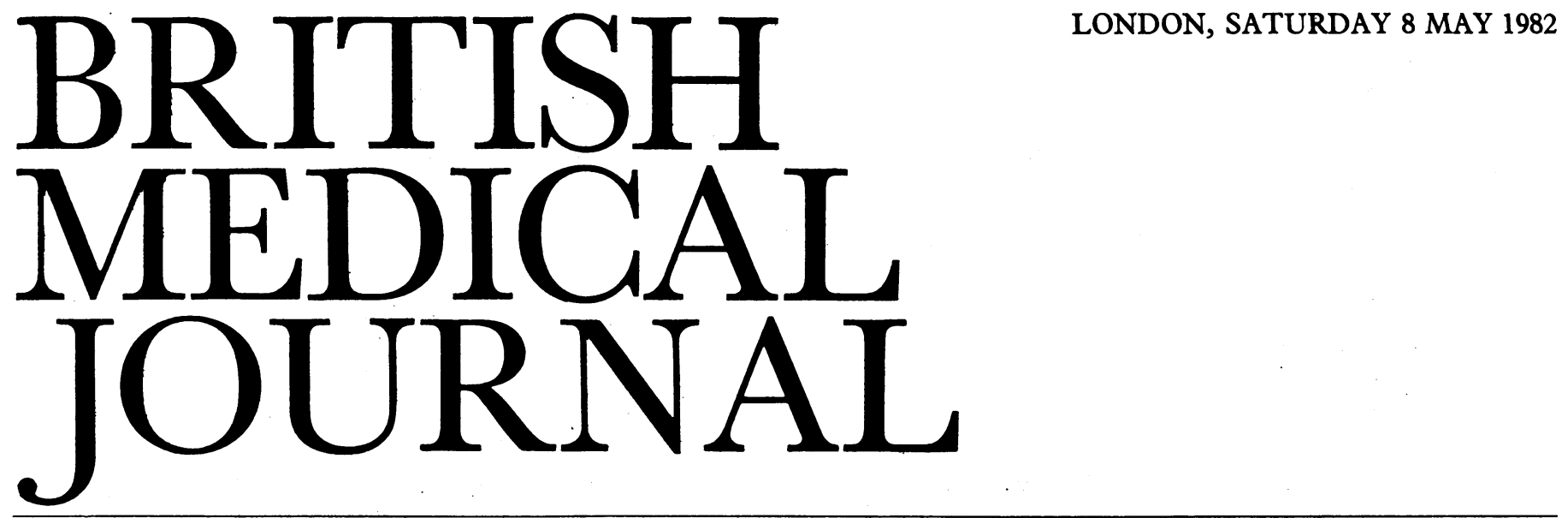

\title{
Congenital malformations and blood glucose control in diabetic pregnancy
}

At the start of the NHS, 27 years after the discovery of insulin in 1921, the outlook for diabetic pregnancy was very poor, with a fetal mortality of about $30 \%$. One of the main achievements in the treatment of diabetes since then has been the steady decline of fetal mortality: with improved standards of care and the establishment of joint clinics most large series now report a loss of about $5 \% \cdot{ }^{1-3}$ This is, however, still two to three times higher than the mortality in babies born to normal women. The respiratory distress syndrome has virtually disappeared as a cause of death in infants of diabetic mothers, but congenital malformations occur three to four times more frequently than in babies born to non-diabetics and account for nearly half of the deaths. ${ }^{245}$ Malformations follow the usual pattern, apart from a considerable increase in the very rare abnormalities of the lower spine and legs known as caudal regression syndrome or sacral dysgenesis.

Maternal hyperglycaemia causes measurable effects on the fetus, whose blood glucose concentration closely reflects that of its mother. Poor diabetic control is associated with a raised fetal mortality, and ketoacidosis is often lethal. Maternal hyperglycaemia causes fetal hyperinsulinaemia, and this is responsible - at least in part-for increased deposition of fat in the infant. ${ }^{6} 7$ Delayed lung maturation and resulting respiratory distress syndrome may be a consequence of fetal hyperinsulinaemia. ${ }^{8}$ It also causes neonatal hypoglycaemia, ${ }^{9}$ a wellrecognised condition in the newborn of diabetic mothers and easily treated; and neonatal hyperbilirubinaemia may also be commoner after prolonged maternal hyperglycaemia. ${ }^{9}$ Congenital malformations, especially of the skeleton, are greatly increased in hyperglycaemic rats. ${ }^{10} 11$ Organogenesis in humans occurs during the first eight weeks of pregnancy ${ }^{12}$ : is there any evidence that hyperglycaemia during this period is related to the development of congenital malformations?

Assessment of diabetic control during the first eight weeks of pregnancy has become possible only recently with the measurement of haemoglobin $A_{1}$, which reflects blood glucose concentrations during the previous four to 12 weeks. Despite many difficulties ${ }^{13}$ it remains a useful guide when serial results in individual patients ${ }^{14}$ are taken in conjunction with conventional assessments of control. Frequently very good control of the blood glucose concentrations (and so "good" haemoglobin $A_{1}$ results) is achieved as pregnancy advances, but control in the first trimester is often poor, ${ }^{914}$ as it is in many nonpregnant diabetics treated with insulin.

An association between a raised haemoglobin $A_{1}$ concen- tration in the first trimester (and therefore hyperglycaemia) and development of fetal congenital malformations has been described in sporadic accounts, ${ }^{915}$ and recently a large study from Boston came to the same conclusions. ${ }^{16}$ Unfortunately, this study was retrospective and the findings cannot be regarded as conclusive. Nevertheless, additional evidence from Denmark shows that fetal malformations occur less frequently in welltreated diabetics from major centres compared with those treated elsewhere, ${ }^{5}$ and first-trimester hyperglycaemia does seem likely to be associated with congenital malformations. The outcome of the prospective multicentre study in Britain is now keenly awaited. We need to remember, however, that most women with high haemoglobin $A_{1}$ values in the first trimester bear normal infants, and a raised haemoglobin $A_{1}$ concentration is not an indication for termination of pregnancy.

The problems of pregnancy should be discussed with diabetic women, preferably before they start their families. Clinic notes of young diabetic women should be tagged and clearly marked after advice has been given in order to avoid repetition. Patients should at least be aware that good diabetic control is important throughout the pregnancy, especially during the first few weeks. They should be advised when control is considered adequate to embark on pregnancy. They will need to be taught techniques of home blood glucose monitoring and have their control assessed with measurements of haemoglobin $A_{1}$ values. Some patients benefit from continuous subcutaneous insulin infusion..$^{1718}$ Yet with all this attention diabetic physicians need to remember to discourage an overobsessional approach; patients should not have to feel a sense of guilt if, despite all these measures, a malformed baby is born.

P J WATKINS

Consultant Physician,

Diabetic Clinic,

King's College Hospital,

London SE5 9RS

1 Jervell J, Bjerkedal T, Moe N. Outcome of pregnancies in diabetic mothers in Norway 1967-1976. Diabetologia $1980 ; 18: 131-4$.

${ }^{2}$ Essex NL, Pyke DA. Management of maternal diabetes in pregnancy. In: Sutherland $\mathrm{HW}$, Stowers JM, eds. Carbohydrate metabolism in pregnancy and the newborn. Berlin: Springer-Verlag, 1979:357-68.

${ }^{3}$ Drury MI, Greene AT, Stronge JM. Pregnancy complicated by clinical diabetes mellitus. A study of 600 pregnancies. Obstet Gynecol $1977 ; 49$ : 519-22.

${ }^{4}$ Malins J. Fetal anomalies related to metabolism: the epidemiological approach. In: Sutherland HW, Stowers JM, eds. Carbohydrate metabolism in pregnancy and the newborn. Berlin: Springer-Verlag, 1979 . 229-46. 
5 Pedersen J. Congenital malformations in newborns of diabetic mothers. In: Sutherland HW, Stowers JM, eds. Carbohydrate metabolism in pregnancy and the newborn. Berlin: Springer-Verlag, 1979:264-76.

- Stubbs SM, Leslie RDG, John PN. Fetal macrosomia and maternal diabetic control in pregnancy. Br Med f 1981 ;282:439-40.

7 Whitelaw A. Subcutaneous fat in newborn infants of diabetic mothers: an indication of quality of diabetic control. Lancet $1977 ; \mathrm{i}: 15-8$.

${ }^{8}$ Stubbs WA, Stubbs SM. Hyperinsulinism, diabetes mellitus, and respiratory distress of the newborn: a common link? Lancet 1978;i : 308-9.

- Ylinen K, Raivio K, Teramo K. Haemoglobin $A_{1 c}$ predicts the perinatal outcome in insulin-dependent diabetic pregnancies. $\mathrm{Br} \mathcal{F}$ Obstet Gynaecol $1981 ; 88: 961-7$.

${ }^{10}$ Deuchar EM. Experimental evidence relating fetal abnormalities to diabetes. In: Sutherland HW, Stowers JM, eds. Carbohydrate metabolism in pregnancy and the newborn. Berlin: Springer-Verlag, 1979: 247-63.

11 Baker L, Egler JM, Klein SH, Goldman AS. Meticulous control of diabetes during organogenesis prevents congenital lumbosacral defects in rats. Diabetes $1981 ; 30: 955-9$.

12 Mills JL, Baker L, Goldman AS. Malformations in infants of diabetic mothers occur before the seventh gestational week. Implications for treatment. Diabetes $1979 ; 28: 292-3$.

13 Anonymous. Haemoglobin $\mathrm{A}_{1}$ and diabetes: a reappraisal. $\mathrm{Br} \mathrm{Med} \mathcal{F} 1980$; 281 : 1304-5.

14 Madsen H, Ditzel J, Hansen P, Hahnemann N, Andersen OP, Kjaargaard $\mathrm{J}$-J. Hemoglobin $\mathrm{A}_{1 \mathrm{c}}$ determinations in diabetic pregnancy. Diabetes Care 1981;4:541-6.

${ }^{15}$ Leslie RDG, Pyke DA, John PN, White JM. Haemoglobin $A_{1}$ in diabetic pregnancy. Lancet 1978 ;ii:958-9.

${ }^{16}$ Miller E, Hare JW, Cloherty JP, et al. Elevated maternal hemoglobin $A_{1 c}$ in early pregnancy and major congenital abnormalities in infants of diabetic mothers. $N$ Engl f Med 1981;304:1331-4.

17 Potter JM, Reckless JPD, Cullen DR. Subciseous continuous insulin infusion and control of blood glucose concentration in diabetics in third trimester of pregnancy. Br Med 7 1980;280:1099-101.

18 Rudolph MC, Coustan DR, Sherwin RS, et al. Efficacy of the insulin pump in the home treatment of pregnant diabetics. Diabetes $1981 ; 30$ : 891-5.

\section{Hypotensive agents, beta- blockers, and drug-induced lupus}

Of the long list of drugs that may induce lupus erythematosus, those used in cardiovascular medicine feature prominently. ${ }^{1-13}$ The best known are procainamide and hydralazine, whose use is associated with the production of antinuclear antibodies and, in fewer patients, with the development of arthralgia, pleurisy, and other features of drug-induced lupus. More recently, sporadic case reports have attributed drug-induced lupus to beta-blockers. How serious are these side effects? What is their frequency? What screening tests are required? Do they relate in any way to the more serious "practolol" oculomucocutaneous syndrome? What are the pathogenetic mechanisms? Reasonable answers to the first four questions are now possible. As to pathogenesis, information is emerging on both genetic and immunological aspects of the syndrome.

Despite its similarities to systemic lupus, drug-induced lupus has several differentiating features: notably the older mean age group, the reversibility on withdrawal of the offending drug, the rarity of renal disease, and, usually, the absence of anti-DNA antibodies. ${ }^{1415}$ In most cases the onset is insidious, usually with weeks or even months of discomfort in the joints. Provided the diagnosis is made early the syndrome is benign and the prognosis excellent-indeed, the clinical importance of the syndrome may well have been exaggerated. Hydralazine, for example, is an effective and useful drug which waned in popularity for several years, largely as a result of fears of the lupus syndrome. Early reports described some $5-10 \%$ of patients as suffering from myalgia, arthralgia, and fever together with positive test results for antinuclear anti- bodies. ${ }^{1}$ Nevertheless, it became apparent that toxicity was partly dose related (earlier usage included doses of up to three $\underline{\underline{\sigma}}$ grams daily) and that other factors (acetylator state, female sex, $\vec{z}$ and possibly genetic factors) were important.

In the study by Mansilla-Tinoco et al $^{16}$ the incidence of $c$ positive antinuclear antibody test results and clinical side effects $\widehat{\widehat{\cong}}$ has been reappraised in patients taking current doses (up to 200-300 mg daily). Antinuclear antibody tests were performed with rat liver substrate with a "screening" dilution of $1 / 20 m$ (several workshops have highlighted the importance of the? methods used). The proportion of positive antinuclear antibody $\overrightarrow{\vec{F}}$ determinations at three years reached $50 \%$ in slow acetylators and $46 \%$ in rapid acetylators. Of 221 patients studied, seven $\frac{C}{0}$ developed clinical features suggestive of drug-induced lupus. In these patients as well as in further referred cases of drug- $\Phi$ induced lupus antinuclear antibody titres were positive at levels of one in 256 or over. All symptoms regressed on stopping the drug.

Thus, with hydralazine at least, positive antinuclear antibody $\overrightarrow{\vec{\omega}}$ test results far outnumber clinical side effects. Conversely, $\frac{\mathscr{D}}{O}$ arthritis and other features of drug-induced lupus seem. extremely unlikely in patients with negative or weakly positive ${ }_{\infty}^{i}$ antinuclear antibody test results. With the use of antinuclear + antibody determinations the practical problems of druginduced lupus on hydralazine may therefore be kept to a? minimum: a rising antinuclear antibody titre provides an $\vec{\omega}$ "early warning" but not necessarily a reason for withdrawing the drug in the absence of symptoms.

The first beta-adrenoceptor-blocking agent reported as inducing the lupus-like syndrome was practolol. ${ }^{6}$ Subsequently, stimulation of antinuclear antibody production or lupus-like $\overrightarrow{ }$ syndromes, or both, have been reported with acebutolol, labetalol, pindolol, and propranolol. ${ }^{7-11}$ If published case ${ }^{\text {N }}$ reports and adverse reports to the Committee on Safety of Medicines are any indication the incidence of drug-induced lupus is extremely rare in relation to the number of prescriptions for beta-blockers. Nevertheless, because the unwitting continuation of treatment generally leads to an increase in the severity of the disease in drug-induced lupus, the results of prospective studies of the incidence of symptoms and of antinuclear antibody conversion, now in progress in Europe, America, and New Zealand, warrant wide publicity.

Does the drug-induced lupus syndrome bear any relation to the practolol oculomucocutaneous syndrome? On the accumulated evidence of the past five years the answer is clearly no. While antinuclear antibodies were originally associated with theo "practolol" syndrome, subsequent studies have shown that the potentially fatal oculomucocutaneous syndrome is a separate음 entity from the more benign and reversible drug-induced lupus $N$ syndrome. Indeed, most patients with the fibrosing practolol $>$ syndrome had negative test results for antinuclear antibodies. ${ }^{13} \stackrel{\mathrm{O}}{=}$ Despite the publicity and the intensely keen observations since, 0 no case of practolol-like toxicity has been convincingly attributed to any other beta-adrenoceptor-blocking agent.

Drug-induced lupus has proved a tempting model fore studying lupus in general. Though antinuclear antibodies, $\mathbb{\Phi}$ usually in high titre, are a feature of drug-induced lupus ${ }^{+}$ (except in rare cases of penicillamine and captopril-induced $\frac{0}{0}$ lupus), anti-DNA antibodies are notably absent. ${ }^{14}{ }^{15}$ Recently Fritzler and $\operatorname{Tan}^{17}$ have identified the nuclear substrate as $a \mathbb{\Phi}^{\circ}$ group of histones. The antibodies are non-complement fixing, $\stackrel{\varrho}{0}$ which may in part explain the low incidence of renal lesions. Advances in nuclear immunochemistry are almost certain too disclose differing antihistone "profiles" for various drugs.

On a more clinical note, genetic susceptibility has long been 